\title{
A knowledgebase to promote self-directed learning among postgraduate trainees of MSc in Biomedical Informatics degree program in University of Colombo, Sri Lanka
}

\author{
G.D.T. Kanchana ${ }^{1 *}$, R.B. Marasinghe ${ }^{2}$ \\ 1 Postgraduate Institute of Medicine, University of Colombo, Sri Lanka \\ 2 Department of Medical Education, University of Sri Jayewardenepura, Sri Lanka \\ *thushari.kanchana@gmail.com \\ https://orcid.org/0000-0001-5509-2393
}

\begin{abstract}
Introduction: Biomedical Informatics degree program was formulated by the Postgraduate Institute of Medicine, University of Colombo to develop medical professionals with digital health competencies to help the digital reforms in Sri Lankan health sector. Medical professionals are expected to have lifelong learning for professional development and for being up to date where Self-Directed Learning (SDL) is considered pivotal. Knowledgebase which includes document repository and discussion forum provides the platform for improving SDL related to biomedical informatics body of knowledge, facilitating collaborative learning and allowing long term knowledge management.
\end{abstract}

Methods: Participatory action research was conducted among the postgraduate (Pg.) trainees who currently enrolled and completed the biomedical informatics degree program. Knowledgebase, a web-based SDL platform using Flask (Python) micro framework was introduced as the intervention and its development was guided by the ADDIE (Analysis, Design, Development, Implementation and Evaluation) model. Validated questionnaires were used as data collection tools. The analysis was done with the pre-implementation stage questionnaire by assessing the SDL practices and requirements for the knowledgebase. The evaluation was done on the knowledgebase users with post-implementation stage questionnaire including the Self Directed Learning Readiness Scale (SDLRS) by Fisher et al. SDLR was assessed along with the improvement of SDL and the relationship of socio-demographics with SDLRS scores. Descriptive and inferential statistics were used for data analysis.

Results: Ninety per cent out of 52 participants confirmed the need for a web-based SDL platform at the pre implementation stage. Internet and peers were the main resources used for SDL. Twenty-three (response rate $=82 \%$ ) knowledgebase users participated in the post implementation stage. Nineteen (82\%) confirmed that the knowledgebase improved their SDL. Mean SDLRS score was 153.83 with highest for self-control (58.52) and lowest for self-management (45.74) subscales. Around 65\% had high SDLRS scores (above 150 out of 200). Statistically significant $(p<0.05)$ relationship existed only between age and SDLRS scores at Mann-Whitney $U$ test, out of the variables under the study.

Conclusions: High level of SDLR was present among the majority of the knowledgebase users which was known to enhance web-based learning. Knowledgebase platform was perceived to improve the SDL among the Pg. trainees, therefore, web-based learning will augment SDL. Attention should be paid to improve selfmanagement among the Pg. trainees of the biomedical degree program to further improve SDL.

Keywords: SDL, Knowledgebase, Web-based learning, Biomedical Informatics 